8

\title{
A Long-term Planning Approach for Small-medium Enterprise Networks
}

\author{
F. Bonfatti, P. D. Monari, R. Montanari, A. Morini \\ Department of Engineering Sciences - University of Modena \\ Via Campi 213/B - 41100 Modena (Italy) \\ tel +3959378514, fax +3959378515 \\ e-mail bonfatti@unimo.it
}

\begin{abstract}
The paper presents the long-term planning approach which has been studied to support small-medium enterprise networks of competing nodes in evaluating their capability to meet the foreseen customer demand. The network as a whole uses long-term planning to identify possible resource lacks in some productive phases and to plan critical materials purchase. The participating nodes use long-term planning to decide the most convenient amount of productive capacity they make available to the network. The planning functions keep demand uncertainty in due consideration and adopt a proper family-based network process model.
\end{abstract}

\section{Keywords}

Enterprise Network Strategies, Long-term Planning, SME Network, Distributed Manufacturing.

\section{$1 \quad$ INTRODUCTION}

Long-term (strategic) planning is a very important support tool for manufacturing enterprises. The effects of foreseen demand configurations are simulated to derive timely estimates of purchasing volumes and times, and of resources workloads (Gessner, 1986). A good long-term plan gives hints to arrange convenient open contracts with suppliers, deferring to medium-term planning the details on product types and volumes. Moreover, it identifies the needs of internal and external productive resources and helps deciding on investments to undertake for improving the enterprise productive capacity.

Unfortunately, small-medium sized enterprises (SMEs) are seldom in condition to employ long-term planning techniques. The reasons are many, in particular: 
- Strategic planning functions, often named Master Production Schedulers (IBM, 1985), are included in costly and burdensome MRP II packages.

- SMEs adopt a lean work organization which cannot afford diverting human resources from directly productive activities to planning activities.

- SMEs frequently apply subcontracting for the execution of process phases, hence these phases are objectively withdrawn from planning.

New organizational paradigms, leading to the constitution of SME networks, are gaining increasing relevance. One of them, addressed to discrete production, is studied within the project EP20723 - PLENT (Planning Small-medium Enterprise Networks) of the European Esprit R\&D program (Bonfatti, 1995).

According to this model: (i) every node can participate in more than one network and maintain its own direct customers; (ii) network nodes have equal rights, although their roles are affected by the declared capacity and the proven reliability; (iii) every node is free in declaring the phases it can perform and the amount of productive capacity it makes available to the network for every phase; (iv) the network takes advantage of the competition of different nodes on the same productive phase; (v) workload distribution among competitor nodes is made on the basis of objective rules that represent the network management policy; (vi) every node is autonomous in deciding investments to improve its own position with respect to competitors within the network.

This organization calls for the introduction of a network coordinating unit (Bonfatti, 1996, 1997). Its capability of applying neutrally the decision and evaluation policy agreed upon by the network nodes, without assuming independent decision powers, is a necessary condition for overcoming the historical distrusts between enterprises, often belonging to the same industrial district, traditionally in competition with each other.

Co-ordinating unit functions are: (i) manage the network process representation in terms of the macro-phases which can be carried out by the different nodes; (ii) manage the representation of work capacities, current states and past performances of the different nodes, with respect to each macro-phase; (iii) manage customer orders, and decide their subdivision into balanced orders to the network nodes so as to pursue node satisfaction and network efficiency; (iv) send orders to the respective nodes, informing them on their position in that specific productive chain (for each node, which are the supplier and the client nodes); (v) arrange new workload distributions in case of problems that cannot be damped down within the network, taking into account node counter-proposals.

With this organization the network can afford design and production of complex products which are out of reach of the single enterprise. Moreover, the network can accept large volume orders thus accessing market segments which otherwise would be precluded. Last but not least, the network can invest in advanced planning techniques, including strategic planning packages. The information coming from long-term planning is relevant for both the network as a whole and the single nodes, as it regards the evaluation of their respective capabilities of meeting the expected customer demand: 
- For the network, long-term planning is used to identify possible lacks of resources on some phases, so as to ask in time the existing nodes to increase their productive capacity, or even to involve new nodes.

- For the single node, long-term planning gives hints to decide on the benefits that could come from modifying its involvement in the network in terms of the subset of phases it performs and the relative declared capacity.

These peculiar goals make the traditional strategic planning techniques unsuitable for enterprise networks. In fact, they adopt process models which are not conceived to represent distributed manufacturing. Moreover, they are focused on estimating material requirements (Volkens, 1995) while for an SME network it is more important to evaluate the overall workload and the effects of different workload distribution policies among nodes. In other words, the specific relationships within an SME network and the particular answers of the involved entities call for the definition of an indeed new approach to long-term planning.

It is the aim of this paper to describe the solution which is studied within the PLENT project. The proposed technique is split into an initial demand definition step and two following planning functions, namely the network plan generation and the nodes plan generation:

- The network plan generation function computes network workloads per phase, and possible critical materials requirements, with respect to the hypothesized external demand, starting from a proper representation of the manufacturing phases which realize the network processes.

- The nodes plan generation function is instead aimed at simulating alternative distributions of the nodes workloads, taking into account their declared productive capacities and according to different network policies.

Either function can be executed autonomously and repeatedly to study different hypothesized scenarios: essentially, demand scenarios for the former and node capacity scenarios for the latter. The intrinsic demand uncertainty is expressed according to a family-based process representation which includes alternative productive paths and the corresponding variations of times and workloads.

The paper presents four main sections. Section 2 introduces the process model supporting the planning functions. Section 3 describes the external demand definition. Finally, Section 4 and Section 5 present and discuss the network plan generation function and the nodes plan generation function, respectively.

\section{2}

\section{THE PROCESS MODEL}

Long-term planning requires to model the network manufacturing processes and the activities that each node can carry out. Considering the planning goals, a high level of detail is not useful. The most natural choice is decomposing every manufacturing process into phases obtained by intersection of the nodes declared activities. The manufacturing process of all the variants of a product family can conveniently be described as a unified direct acyclic graph (DAG), called network 
operational scheme, which includes alternative production phases and paths. The operational scheme syntax is based on the graphical primitives shown in Figure 1.

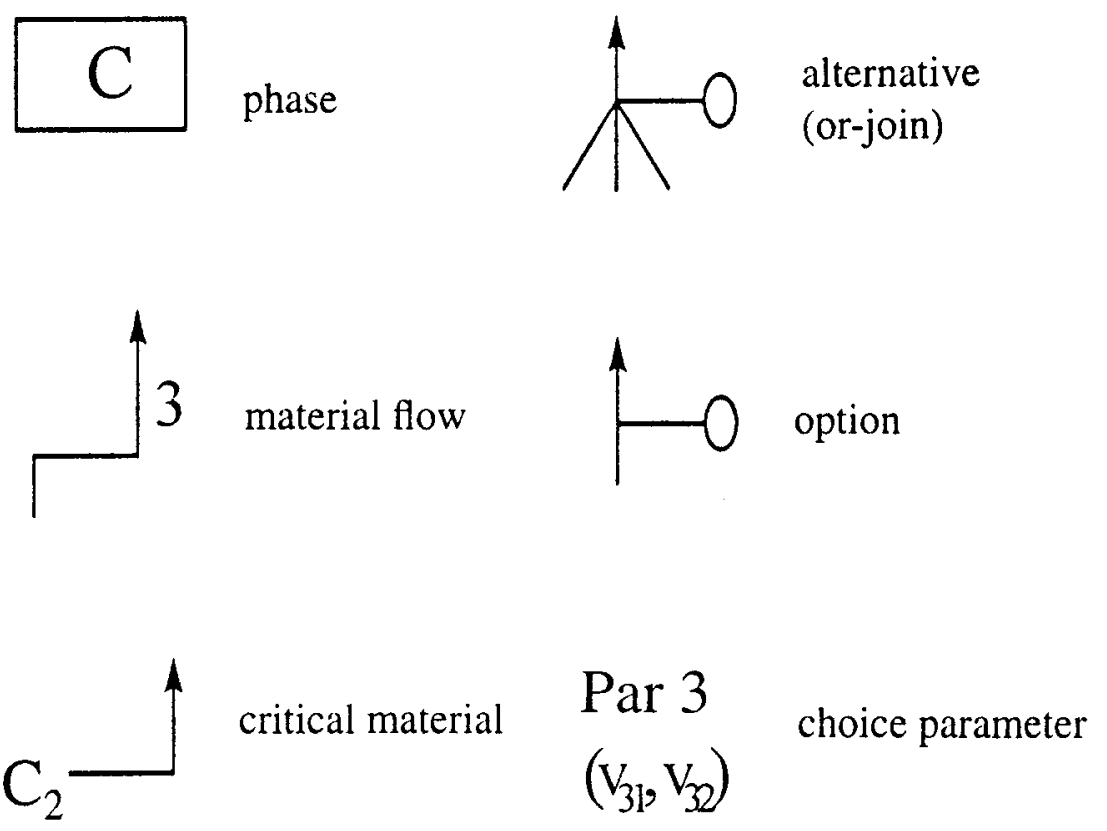

Figure 1 Primitives of the network operational scheme.

\section{Phase}

We define network process phase every sequence of manufacturing operations, no matter how complex, which can be executed by one or more network nodes without interruptions. If node $\mathrm{Nl}$ can execute a given sequence of operations and node $\mathrm{N} 2$ can execute only a part of it, the N1 activity must be split into three phases, one of which is executed by either nodes. Nodes performances with respect to a given phase differ in execution time and productive capacity.

\section{Material flow}

Oriented arcs represent the flows of materials between the phases which produce them and those which use them. When more than one phase uses the same material, as many flow lines leave the phase producing it. Every arc has associated the corresponding multiplying factor, a positive integer number expressing the quantity of input material required to realize a unit of output material. The absence of this number means that a unitary factor is applied. 


\section{Critical material}

This primitive represents the raw material or component whose purchasing is in charge of the network as a whole (components purchased by the single node are not considered to preserve the autonomy of its behavior). The represented critical materials are visible to the network planning function so as to properly estimate their requirements over time. Also critical materials are characterized by quantity multiplying factors.

\section{Alternative}

Each product variant has its own operational scheme, but often it shares most phases with other products of the same family. Moreover, a given component may be obtained following alternative, interchangeable production paths which, leading to the same result, do not impact on the subsequent phases. The alternative primitive represents either situations (Bonfatti, 1998) and avoids the redundancy which could result from writing a different scheme for every variant of the network final product.

\section{Option}

A particular case of alternative is given by the possibility of choosing between executing or skipping a certain phase. These phases correspond to manufacturing activities of optional components or auxiliary tasks required under particular circumstances. For instance, the request of a customized product sometimes involves the execution of a design phase which is otherwise unnecessary.

\section{Choice parameter}

Both the above alternative types have associated choice parameters with as many values as the number of options. The same parameter can be associated to more than one operational scheme alternatives, provided that they present the same number of options, to denote that those alternatives are subject to the same choice criterion. A particular combination of parameter values identifies a sub-family or a single variant. This mechanism is used in external demand definition. 


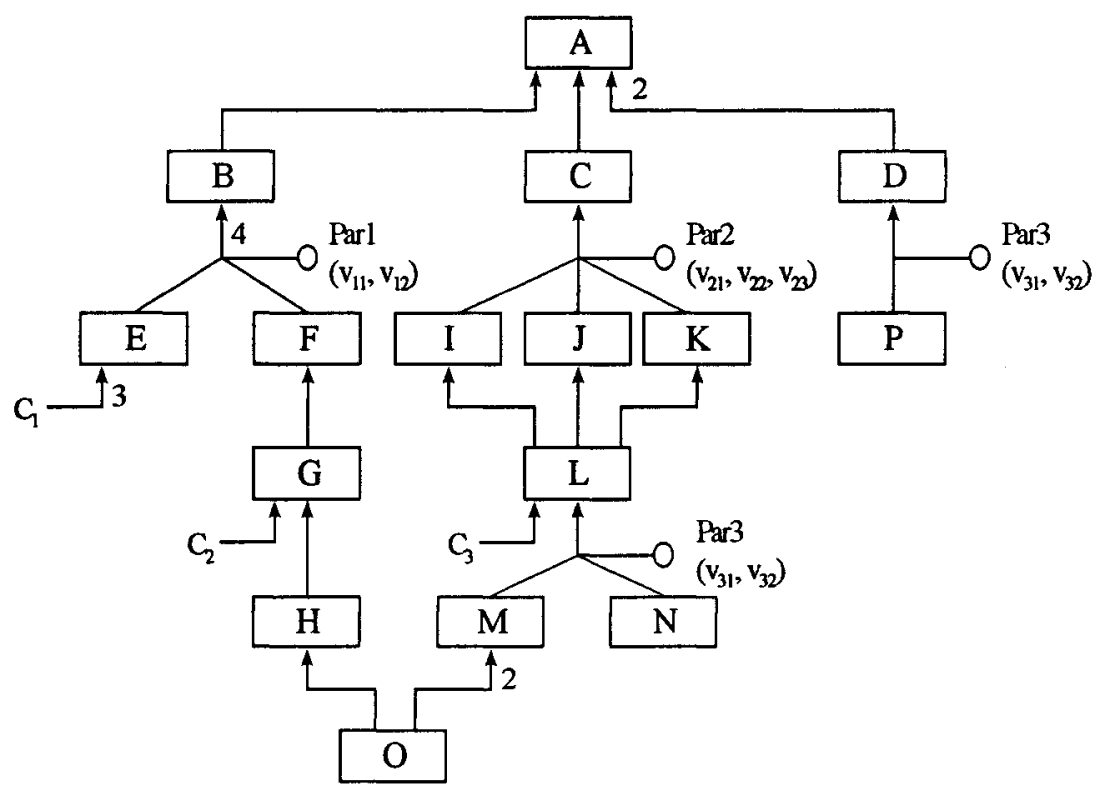

Figure 2 Example of network operational scheme.

Figure 2 reports an example of operational scheme. In case of discrete production, the graph root (phase $\mathrm{A}$ in the example) is unique and corresponds to the last process phase, for instance final assembly or packaging and delivery. Leaves (phases $\mathrm{E}, \mathrm{O}, \mathrm{N}$ and $\mathrm{P}$ ) are many and indicate the initial manufacturing phases which realize the simplest semi-finished products.

The operational scheme in Figure 2 shows situations that can be recognized as typical cases of workflow management, as they are defined in the WfMC reference model (Hollingsworth, 1995). A case of and-split corresponds to the flows leaving phase $O$ to feed in parallel two successive independent phases. The flow leaving phase $\mathrm{L}$ is an or-split as it implies, when executing a given order instance, the choice of one of the possibilities leading to phase I, J or K, alternatively. An and-join case is constituted by the material flows entering phase A and expresses the simultaneous use of three input materials (two particular andjoin occurrences are also given by the flows entering phases $G$ and $L$ ). Finally, three or-join cases realize the convergence of the alternative paths leading to phases $\mathrm{B}, \mathrm{C}$ and $\mathrm{L}$.

Observe that, in the example of Figure 1, parameter Par3 is associated to both the alternative leading to phase $\mathrm{L}$ and the option leading to phase $\mathrm{D}$. This means that the values assigned to the choice parameter options affect both manufacturing paths although they belong to independent branches of the operational scheme. 
Node capacity per phase

The process model is completed by the declaration of the productive capacities that each node makes available to the network with respect to the phases it can execute. Taking into account the long time horizon of strategic planning, we assume the day as time unit. Then, every pair (node, phase) has associated the daily capacity, $\mathrm{C}_{d}$, expressing the average number of output material units produced every day. We suppose that the productive capacities of the node are not concurrent, i.e., they are independent from each other.

\section{3}

\section{DEMAND REPRESENTATION}

The first step of the planning procedure is aimed at declaring the demand configuration that represents the long-term production budget. Starting from the sales forecasts provided by the marketing function, a set of external demand items, properly distributed along the planning horizon, are issued. Each item represents the total requirement on a given period for a certain family or sub-family of products. We consider sufficient to express the requirement temporal granularity on a weekly basis. The demand item presents the following structure:

\begin{tabular}{lllll}
\hline Year & Week & Family & Quantity & Configuration \\
\hline 1997 & 11 & Fl & 200 & $\operatorname{Parl}\left(\mathrm{v}_{11}=40, \mathrm{v}_{12}=60\right)$ \\
& & & & $\operatorname{Par}\left(\mathrm{v}_{21}=0, \mathrm{v}_{22}=100, \mathrm{v}_{23}=0\right)$ \\
& & & $\operatorname{Par3}\left(\mathrm{v}_{31}=25, \mathrm{v}_{32}=75\right)$ \\
\hline
\end{tabular}

- Year and Week express the due date of the requirement instance.

- Family and Quantity indicate, respectively, the type of product to plan and the number of pieces of the requirement instance.

- Configuration is made of as many expressions as the family choice parameters: it is aimed at representing the distribution of product variants within the considered family. Every parameter must be instantiated, each option must receive a value and the sum of these values must always be 100 .

It is worth observing how this representation of the requirement instance configuration can characterize every possible type of demand. If one or more parameters have specified just one option with value 100 (as for parameter Par2) then the demand item refers to a sub-family where the choices corresponding to the zero value options are excluded. Moreover, if all the family parameters present the 100 choice, the demand item refers to a single family variant.

We call network planning the computation of phases workloads and critical materials requirements as they results with respect to the hypothesized external 
demand. The planning algorithm works at infinite capacity, hence it can process one demand item at a time. The operational scheme of the single demand item is examined backward (from root to leaves) to calculate quantities of output materials and duration of each phase. In correspondence of the alternatives the computation considers the different paths and the rates associated to each of them. Figure 3 shows the requirements of output and critical materials resulting for the operational scheme of Figure 2 in case of a demand item of 100 pieces, and according to the parameter values reported in legend.

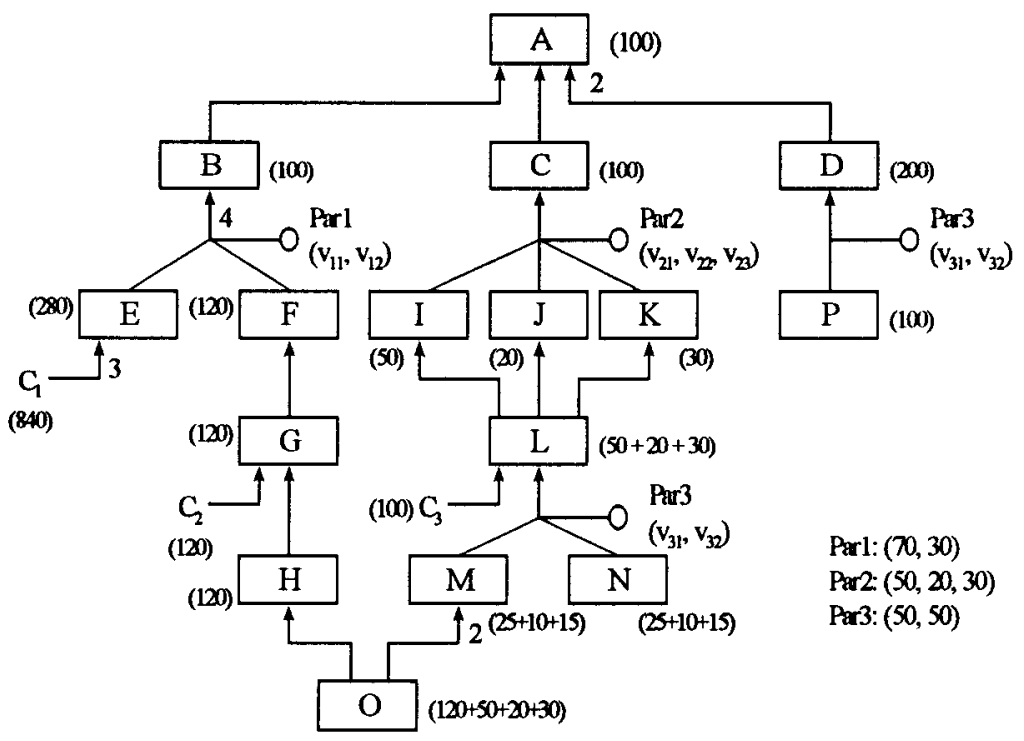

Figure 3Materials requirements per phase.

For the execution of the network planning function we choose the most efficient workload distribution among the network nodes, i.e., the distribution which minimizes the lead-times. The main aim we pursue with network planning is in fact determining the minimal execution time to adopt as a reference datum for evaluating the criticality of the network behavior. Since every phase can be carried out by a number of competing nodes, its duration may change considerably depending on the actual workload distribution.

In order to determine the distribution which minimizes the phase execution time, let us denote with $\mathrm{BS}_{\mathrm{j}}$ the output batch size of the $\mathrm{j}$-th phase $\left(\mathrm{BS}_{\mathrm{j}}\right.$ is a function of the quantities consumed by the following phases) and suppose it can be executed by $\mathrm{n}$ nodes. The distribution is obtained by solving the following system of $(n+1)$ linear equations: 


$$
\begin{aligned}
& T_{j}=\frac{x_{i j}}{c_{i j}} \quad i=1 . n \\
& \sum_{i=1}^{n} x_{i j}=B S_{j}
\end{aligned}
$$

where $x_{1 j}, x_{2 j}, \ldots x_{n j}$ express the number of pieces produced by each of the $n$ nodes, $c_{i j}$ the capacity of node $i$ on phase $j, T_{j}$ the minimal execution time.

For example, suppose that three enterprises declare to perform the $j$-th phase with a productive capacity of 10,40 and 100 pieces per day, respectively. If it is $\mathrm{BS}_{\mathrm{j}}=400$, the solutions are $\mathrm{x}_{\mathrm{j} 1}=26.66, \mathrm{x}_{\mathrm{j} 2}=106.66, \mathrm{x}_{\mathrm{j} 3}=266.6$ with $\mathrm{T}_{\mathrm{j}}=2.66$. Since workloads must be expressed by integer numbers, the three nodes will have assigned 26, 107 and 267 pieces, respectively. The actual phase duration coincides with the time spent by the slowest node: $T_{j 2}=107 / 40=2.75$ days (here, the second $T$ index identifies the node). It means that the phase lasts for three days: in two of them the nodes work at their maximum capacity while in the third day only a fraction of the capacity is actually spent.

The start date of the $j$-th phase is finally computed by subtracting the $T_{j}$ duration from the phase due date. Whenever a phase must feed two or more following phases, these issue independent requirements which are managed autonomously. In the example of Figure 3 this situation is represented by lists of batches associated to phases $\mathrm{L}$ and $\mathrm{O}$ (and phases $\mathrm{M}$ and $\mathrm{N}$ as they precede $\mathrm{L}$ ).

Once the network planning function has been applied to all the demand items, resource profiles such as that reported in Figure 4 give a measure of the global capacity and the capacity peaks the network must for every phase. 


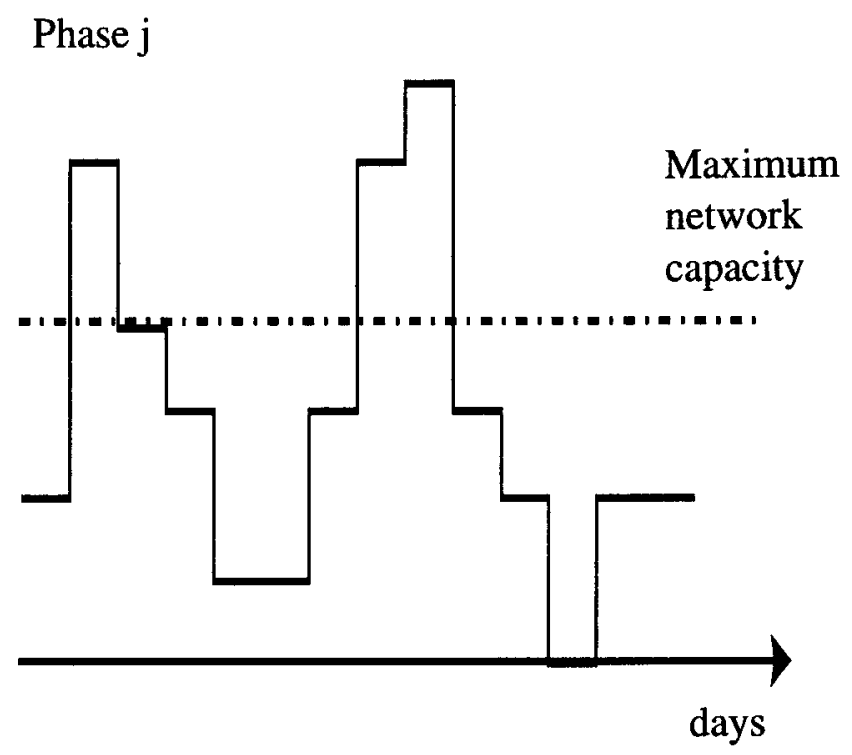

Figure 4Resource profile for the $j$-th phase.

\section{NODES PLAN GENERATION}

The node workloads planning function is executed to evaluate which task assignments every node may expect to receive with reference to the hypothesized demand. The computation is carried out at finite capacity so as to take into account the concurrence of demand items on the productive capacities. The planning algorithm realizes a simplified simulation of the network policy: it tries to balance the ratio between the tasks assigned to the different nodes and their declared capacities by taking also into account the rank attributed to each node.

The planning algorithm examines one demand item at a time. The demand items are examined from the nearest week to the most distant future. Within a week, the examination order is decided by the planner. Assignment of demand item phases to nodes is carried out according to a backward approach and each phase is assigned to a single node, without introducing batch splittings. For each phase, workload assignment to nodes is executed on a daily basis: starting from the phase due date the algorithm determines the node that is more entitled to receive the assignment. If the daily available capacity is not sufficient to run out the batch 
size, the selected node is charged for the needed number of days. The node daily capacity is decreased according to each received assignment.

Whenever two or more nodes are able to perform a phase, a dynamic node priority is used to decide which node has the right to obtain the assignment. The priority of the $\mathrm{i}$-th node on the $\mathrm{j}$-th phase is given by the expression:

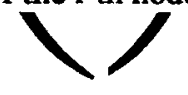

$$
\text { if } \mathbf{a}_{\mathbf{i}}>0
$$

$$
\text { if } a_{i}=0
$$

where:

- $\mathrm{Ri}$ is a coefficient ranging between 0.0 and 1.0 which expresses the node rank (this coefficient expresses node reliability as it results from the evaluation of its past performances);

- $\mathrm{x}_{\mathrm{ijk}}$ represents the number of pieces assigned to node $\mathrm{i}$ on phase $\mathrm{j}$ for the $\mathrm{k}$-th day ( $\mathrm{d}$ is the due date of the demand item under examination);

- $c_{i j}$ represents the productive capacity declared by node $i$ for phase $j$

- $\mathrm{a}_{\mathrm{j}}=\operatorname{card}\left\{\mathrm{k} \mid \exists \mathrm{i}: \mathrm{x}_{\mathrm{ijk}} \neq 0\right\}$ counts the number of days where at least one node has received an assignment for phase $\mathrm{j}$.

As an example, consider three nodes $\alpha, \beta$ and $\gamma$ which declare respectively a capacity of 40,20 and 60 pieces per day for phase j. Suppose that the requirements for phase j are: (i) 50 pieces on day 96, (ii) 200 pieces on day 98 and (iii) 100 pieces on day 99 and that the following plan constitutes the situation as it results

\begin{tabular}{|c|c|}
\hline \multicolumn{2}{|r|}{$c_{i j}$} \\
\hline$\alpha$ & 40 \\
\hline$\beta$ & 20 \\
\hline$\gamma$ & 60 \\
\hline
\end{tabular}
from the previous assignments:

\begin{tabular}{|c|c|c|c|c|c|c|c|c|}
\hline & 92 & 93 & 94 & 95 & 96 & 97 & 98 & 99 \\
\hline$\ldots$ & & & & & & & & \\
\hline$\ldots$ & 10 & 20 & & & & & & \\
\hline$\ldots$ & & & 30 & & & & & \\
\hline
\end{tabular}

$$
\text { days }
$$

Assuming $R_{i}=1$ for every node, the priorities result: $P_{\alpha j}=1, P_{\beta i}=0.5, P_{\gamma j}=0.83$. Then the (i) batch is assigned to node $\alpha$, more precisely 40 pieces on day 95 and the remaining 10 pieces on day 94 . The new priorities become: $\mathrm{P}_{\alpha \mathrm{j}}=0.69, \mathrm{P}_{\beta \mathrm{i}}=$ $0.62, P_{\gamma j}=0.88$. Thus the (ii) batch is assigned to node $\gamma$ on days 97 to 94 . Finally, the priorities change again: $P_{\alpha j}=0.79, P_{\beta i}=0.75, P_{\gamma j}=0.36$ and the (iii) batch is assigned to node $\alpha$, on days 98 to 96 . The resulting plan is now:

\begin{tabular}{|l|l|}
\multicolumn{1}{l|}{} & $\mathrm{c}_{\mathrm{ij}}$ \\
\hline$\alpha$ & 40 \\
\hline$\beta$ & 20 \\
\hline$\gamma$ & 60 \\
\hline
\end{tabular}

days \begin{tabular}{c|c|c|c|c|c|c|c|c|}
\multicolumn{2}{c}{92} & 93 & 94 & 95 & 96 & 97 & 98 & 99 \\
\hline$\ldots$ & & & 10 & 40 & 20 & 40 & 40 & \\
\hline$\ldots$ & 10 & 20 & & & & & & \\
\hline$\ldots$ & & & 50 & 60 & 60 & 60 & & \\
\hline
\end{tabular}


and the corresponding priorities are $P_{\alpha j}=0.46, P_{\beta i}=0.79, P_{\gamma j}=0.45$. It means that the next batch (if any) will be assigned to node $\beta$.

In conclusion, observe that this finite capacity workload distribution can be executed many times changing the node priorities (i.e. their rank and declared capacities) to evaluate different planning scenarios. These results, together with the network resource profiles, are made available to the network nodes to support them in deciding their own strategy and the network general policy.

This approach is currently under validation at four enterprises networks: (i) an Italian network manufacturing a family of wheel-motors; (ii) a Spanish network manufacturing complex transfer machines; (iii) a Greek network producing garments; (iv) an Hungarian network producing oil for food industry.

Bonfatti, F. and Monari, P.D. (1995) Planning small-medium enterprise networks. Proc. of the Int. Conf. IMPDE '95 on Improving Manufacturing Performance in a Distributed Enterprise, Edinburgh.

Bonfatti, F., Monari, P.D. and Paganelli, P. (1996) Co-ordination functions in SME networks, in Balanced Automation Systems II (ed. L.M. Camarinha-Matos, H. Afsarmanesh), Chapman \& Hall, London.

Bonfatti, F., Monari, P.D. and Montanari, R. (1997) Information flows and processes in a SME network. Proc. of the OE/IEEE/IFIP Int. Conf. ISIP '97 on Integrated and Sustainable Industrial Production, Lisbon.

Bonfatti, F., Monari, P.D. and Paganelli, P. (1998) Resource-free and resourcedependent aspects of process modeling: a rule-based conceptual approach. Int. Journal of Computer Integrated Manufacturing (IJCIM), Taylor \& Francis, London.

Gessner, R.A. (1986) Master Production Schedule Planning. John Wiley \& Sons, New York.

IBM (1985) Master Production Schedule Planning Reference Manual. Atlanta.

Hollingsworth, D (1995) The Workflow Reference Model. The Workflow Management Coalition, Brussels.

Volkens, B. (1985) Planning bills for master production scheduling. Proc. of the APICS 28th Int. Conf., Toronto. 
Flavio Bonfatti. Graduated in Electronics Engineering. Associated professor of Fundamentals of Computer Science and lecturer of Software Engineering. Scientific responsible of a number of national and international research projects.

Paola Daniela Monari. Graduated in Mathematics. Consultant in manufacturing information systems and industrial applications and lecturer of Industrial Informatics. Involved in several national and international research projects.

Roberto Montanari. Graduated in Informatics Engineering. PhD student in software engineering for industrial applications.

Andrea Morini. Graduated in Informatics Engineering. Researcher in product modeling and production planning. Involved in international research projects. 